\title{
Aplicación de la simulación discreta para proponer mejoras en los procesos de atención en el área de emergencia de un hospital público
}

\section{RESUMEN}

En esta investigación se ha evaluado la situación actual del área de emergencia que atiende adultos, en un hospital público del Perú. Se utilizó el software Arena para realizar simulaciones discretas, con el objetivo de obtener propuestas de mejora que permitan reducir el tiempo total de permanencia del paciente en esta área antes de que sea dado de alta 0 admitido en el hospital.

Se desarrolló un modelo de simulación donde se identificaron varios tipos de pacientes que se atendieron en los tópicos de cirugía, medicina y traumatología. En algunos casos se requirieron análisis en laboratorios.

Para disminuir el tiempo de permanencia de un paciente en el sistema se evaluaron diversas posibilidades, y se obtuvo como mejor alternativa la modificación en el horario de atención de los médicos en el tópico de medicina. Esta solución considera que cuatro médicos atiendan de 7:00 a.m. a 7:00 p.m. y dos médicos de 7:00 p.m. a 7:00 a.m.

Palabras clave: Simulación, Hospital, Programación.

DISCRETE SIMULATION APPLICATION FOR DEVELOPING IMPROVEMENTS FOR THE PROCESSES OF THE EMERGENCY SERVICE DEPARTMENT OF A PUBLIC HOSPITAL

\section{ABSTRACT}

In this investigation it has been evaluated the current situation of the emergency area that assists adults, in a public hospital of Peru. The software Arena was used to perform discreet simulations, with the objective of obtaining improvement proposals that allow reduce the total time of the patient's permanency in this area before he is to discharge from hospital or admitted in the hospital.

A simulation model was developed where several types of patients were identified that were assisted in the surgery, medicine, and traumatología topics. In some cases clinical analysis was required.

For reduce the time of a patient's permanency in the system diverse possibilities were evaluated, being the best alternative a modification in the schedule of the doctors' attention in the medicine topic. This solution considers that four doctors assist from 7:00 a.m. to 7:00 p.m. and two doctors from 7:00 p.m. to 7:00 a.m.

Key words: Simulation, Hospital, Scheduling.

\section{INTRODUCCIÓN}

Los servicios de salud en el Perú se brindan a través de los sectores privado y público, este último sector concentra el $51 \%$ de los hospitales, según datos del Ministerio de Salud (MINSA, 2007). El hospital que se estudiará es considerado uno de los más importantes del país, debido a su infraestructura, cantidad de pacientes atendidos en consultorios y en el área de emergencia cada año, por la variedad y complejidad de casos atendidos, siendo posible esto último pues se cuenta con personal médico altamente especializado.

En el hospital en estudio, el área de emergencia es una de las más críticas debido a que está encargada de proporcionar atención médica oportuna y eficiente a todas las personas cuya vida está en riesgo. La atención en esta área se realiza durante las 24 horas del día, todos los días. El área de emergencia tiene cuatro zonas: Emergencia Pediátrica, Emergencia Obstétrica, Salud Mental y Emergencia Adultos, siendo esta última la más concurrida en los últimos años. Por todo lo expuesto, el estudio se centrará en Emergencia Adultos.

Dada la importancia de que en el área de emergencia se brinde a los pacientes una atención rápida, y con el menor tiempo de espera posible, el estudio a realizar evaluará la zona Emergencia Adultos para proponer soluciones que reduzcan el tiempo de permanencia de los pacientes en esta área antes de que lo den de alta o lo deriven al área de internamiento del hospital.

\section{DESCRIPCIÓN DEL SISTEMA ACTUAL}

Un paciente que llega a Emergencia Adultos, que cuenta con ocho salas de atención, recibe una primera evaluación en la sala de Triaje, donde el médico de turno evaluará sus signos vitales, le asignará un nivel de prioridad y lo derivará a una unidad de atención según el tipo de dolencia. A continuación, el paciente debe registrarse en la oficina de Admisión, donde se anotarán sus datos personales y la unidad donde se le derivó.

En Emergencia Adultos los pacientes se clasifican según la gravedad de su enfermedad o dolencia en cuatro niveles de prioridad, que son los siguientes (de mayor a menor): I. Atención

\footnotetext{
Ingeniera Industrial, PUCP, Consultora, ActualiSAP Consultores E-mail: kdelgado@actualisap.com

2 Doctor en Ingeniería Industrial, UNMSM, Profesor del Departamento de Ingeniería, PUCP. E-mail:miguel.mejia@pucp.edu.pe
} 
inmediata por shock y/o trauma, II. Atención antes de los diez minutos, III. Atención después de diez minutos, IV. Atención por consulta externa.

Los pacientes de prioridad I son enviados a la sala de reanimación y luego a unidad de dolor torácico o a la unidad crítica de pacientes, dependiendo del caso. Los pacientes de prioridad II y III pueden ser enviados a tópico de cirugía, tópico de medicina o tópico de traumatología. Después de ser atendidos los pacientes son observados en las salas de reposo o son dados de alta.

En todos los casos, el médico puede indicar que se realice al paciente algún tipo de evaluación especial que requiera el uso del laboratorio, tomografía axial computarizada o rayos $\mathrm{X}$. Una vez solicitada la evaluación correspondiente por el médico, la orden es llevada a la Oficina de Digitación donde se imprime un documento que debe ser presentado en la unidad respectiva. Si se requiere una muestra para el laboratorio, el personal encargado se desplaza al encuentro del paciente, toma la muestra y la lleva al laboratorio para luego recoger los resultados. Si se trata de otro tipo de evaluación se llevará al paciente a las unidades respectivas.

Los pacientes se han clasificado en quince tipos, que se muestran en la Tabla N. ${ }^{0} 1$.

Luego de la evaluación de la sala de Triaje, la probabilidad asociada a la asignación del paciente a un determinado tópico, se muestra en la Tabla N. ${ }^{\circ} 2$. Es- tas probabilidades son resultados del levantamiento de información realizado en Emergencia Adultos.

Tabla N. ${ }^{\circ}$ 2. Probabilidad de que un paciente sea derivado a un tópico o sala.

\begin{tabular}{|l|c|}
\hline \multicolumn{1}{|c|}{ Ambiente } & Probabilidad \\
\hline Sala de Espera & 0.079 \\
\hline Medicina & 0.403 \\
\hline Cirugía & 0.170 \\
\hline Traumatología & 0.152 \\
\hline Shock-Trauma & 0.033 \\
\hline Otros (no emergencia) & 0.163 \\
\hline \multicolumn{2}{|r|}{ TOTAL } \\
\hline
\end{tabular}

Fuente: Hospital público; elaboración propia

A continuación se muestra en la Tabla N ${ }^{\circ} 3$, los porcentajes por tipo de paciente en cada tópico.

Los pacientes tipo 3, 7, 11 y 14 no tienen un porcentaje asignado debido a que ellos son los mismos pacientes que ingresaron por primera vez a un tópico de Emergencia Adultos.

Los pacientes tipo 15 ingresan al sistema con una prioridad I, son atendidos en Triaje y luego su acompañante se dirige a Admisión mientras que el paciente es atendido en el tópico de Shock-Trauma, luego lo derivan al área de internamiento de hospital. Debido a que solo se analizará la zona Emergencia Adultos, se considerará que este tipo de pacientes sale del sistema una vez que fue atendido.

Tabla 1. Clasificación de los pacientes atendidos en Emergencia Adultos

\begin{tabular}{|c|l|l|}
\hline $\begin{array}{c}\text { Tipo de } \\
\text { paciente }\end{array}$ & \multicolumn{1}{|c|}{ Tópico } & \multicolumn{1}{c|}{ Descripción } \\
\hline 1 & Medicina & Paciente de alivio que no necesita una segunda revisión \\
\hline 2 & Medicina & Paciente de alivio que sí necesita una segunda revisión \\
\hline 3 & Medicina & Paciente de alivio que ingresa para su segunda revisión \\
\hline 4 & Medicina & Paciente de medicina sin atención previa que no requiere análisis \\
\hline 5 & Medicina & $\begin{array}{l}\text { Paciente de medicina sin atención previa que requiere análisis en el } \\
\text { laboratorio }\end{array}$ \\
\hline 6 & Medicina & Paciente de medicina sin atención previa que requiere otros análisis \\
\hline 7 & Medicina & Paciente de medicina con resultados de análisis \\
\hline 8 & Cirugía & Paciente de cirugía sin atención previa que no requiere análisis \\
\hline 9 & Cirugía & $\begin{array}{l}\text { Paciente de cirugía sin atención previa que requiere análisis en el } \\
\text { laboratorio }\end{array}$ \\
\hline 10 & Cirugía & Paciente de cirugía sin atención previa que requiere otros análisis \\
\hline 11 & Cirugía & Paciente de cirugía con resultados de análisis \\
\hline 12 & Traumatología & Paciente de traumatología sin atención previa que no requiere rayos X \\
\hline 13 & Traumatología & Paciente de traumatología sin atención previa que requiere rayos X \\
\hline 14 & Traumatología & Paciente de traumatología con resultados de rayos X \\
\hline 15 & Shock-Trauma & Paciente crítico \\
\hline
\end{tabular}

Fuente: Elaboración propia 
Tabla N. ${ }^{\circ}$ 3. Distribución porcentual de los tipos de pacientes atendidos en cada tópico de Emergencia Adultos.

\begin{tabular}{|c|c|c|c|c|c|}
\hline \multicolumn{2}{|c|}{ Tópico de Medicina } & \multicolumn{2}{|c|}{ Tópico de Cirugía } & \multicolumn{2}{|c|}{ Tópico de Traumatología } \\
\hline $\begin{array}{c}\text { Tipo de } \\
\text { paciente }\end{array}$ & Porcentaje & $\begin{array}{c}\text { Tipo de } \\
\text { paciente }\end{array}$ & Porcentaje & $\begin{array}{l}\text { Tipo de } \\
\text { paciente }\end{array}$ & Porcentaje \\
\hline 1 & $20.69 \%$ & 8 & $41.67 \%$ & 12 & $36.67 \%$ \\
\hline 2 & $10.34 \%$ & 9 & $29.17 \%$ & 13 & $63.33 \%$ \\
\hline 4 & $24.14 \%$ & 10 & $29.17 \%$ & TOTAL & $100.00 \%$ \\
\hline 5 & $37.93 \%$ & TOTAL & $100.00 \%$ & & \\
\hline 6 & $6.90 \%$ & & & & \\
\hline TOTAL & $100.00 \%$ & & & & \\
\hline
\end{tabular}

Fuente: Elaboración propia

En la tabla $\mathrm{N} .^{\circ} 4$ se muestran las secuencias de recorrido que sigue cada tipo de paciente. También se indica qué tipos de pacientes se convierten en otro tipo. Así tenemos que para efectos de la simulación, los siguientes casos:

a) El paciente tipo 1 realiza el recorrido Triaje-Admisión-Medicina-Farmacia-Salida.

b) El paciente tipo 2 realiza el recorrido TriajeAdmisión-Medicina-Farmacia-Sala de Espera, y luego el recorrido Medicina-Farmacia-Salida, pero como paciente tipo 3.

c) El paciente tipo 4 realiza el recorrido Triaje-Admisión-Medicina-Farmacia-Salida.

d) El paciente tipo 5 realiza el recorrido TriajeAdmisión-Medicina-Digitación-Análisis en Laboratorio-Otros Análisis, y luego el recorrido Digitación-Medicina-Farmacia-Salida, pero como paciente tipo 7.

e) El paciente tipo 6 realiza el recorrido Triaje-Admisión-Medicina-Digitación-Laboratorio, y luego el recorrido Digitación-Medicina-Farmacia-Salida, pero como paciente tipo 7 .

f) El paciente tipo 8 realiza el recorrido Triaje-Admisión-Cirugía-Farmacia-Salida.

g) El paciente tipo 9 realiza el recorrido TriajeAdmisión-Cirugía- Digitación-Análisis en Laboratorio-Otros Análisis, y luego el recorrido Digitación-Cirugía-Farmacia-Salida, pero como paciente 11.

h) El paciente tipo 10 realiza el recorrido Triaje-Admisión-Cirugía- Digitación-Laboratorio, y luego el recorrido Digitación-Cirugía-Farmacia-Salida, pero como paciente 11 .

i) El paciente tipo 12 realiza el recorrido TriajeAdmisión-Traumatología-Farmacia-Salida.

j) El paciente tipo 13 realiza el recorrido TriajeAdmisión-Traumatología-Digitación-Rayos $X, y$ luego el recorrido Traumatología-Farmacia-Salida, pero como paciente 14 (Tabla 4).

Tabla N. ${ }^{\circ}$ 4. Secuencias de recorrido para cada tipo de paciente.

\begin{tabular}{|c|l|c|}
\hline $\begin{array}{c}\text { Tipo de } \\
\text { paciente }\end{array}$ & \multicolumn{1}{|c|}{ Secuencia de recorrido } & $\begin{array}{c}\text { ¿Nuevo Tipo } \\
\text { de paciente? }\end{array}$ \\
\hline 1 & Triaje-Admisión-Medicina-Farmacia-Salida & No \\
\hline 2 & Triaje-Admisión-Medicina-Farmacia-Sala de Espera & Si, Tipo 3 \\
\hline 3 & Medicina-Farmacia-Salida & No \\
\hline 4 & Triaje-Admisión-Medicina-Farmacia-Salida & No \\
\hline 5 & Triaje-Admisión-Medicina-Digitación-Análisis en Laboratorio-Otros Análisis & Si, Tipo 7 \\
\hline 6 & Triaje-Admisión-Medicina-Digitación-Laboratorio & Si, Tipo 7 \\
\hline 7 & Digitación-Medicina-Farmacia-Salida & No \\
\hline 8 & Triaje-Admisión-Cirugía-Farmacia-Salida & No \\
\hline 9 & Triaje-Admisión-Cirugía--Digitación-Análisis en Laboratorio-Otros Análisis & Si, Tipo 11 \\
\hline 10 & Triaje-Admisión-Cirugía--Digitación-Laboratorio & Si, Tipo 11 \\
\hline 11 & Digitación-Cirugía-Farmacia-Salida & No \\
\hline 12 & Triaje-Admisión-Traumatología-Farmacia-Salida & No \\
\hline 13 & Triaje-Admisión-Traumatología-Digitación-Rayos X & Si, Tipo 14 \\
\hline 14 & Traumatología-Farmacia-Salida & No \\
\hline
\end{tabular}


La Sala de Espera indicada para los pacientes tipo 2 , se ha considerado en el recorrido porque ellos deben esperar un tiempo determinado para que la medicina aplicada les haga efecto; luego puedan ingresar a su segunda evaluación como pacientes tipo 3.

\section{MODELADO DEL SISTEMA}

Para determinar el tamaño de muestra fue necesario realizar una prueba piloto. Esta prueba permitió identificar tres intervalos de tiempos adecuados para la toma de datos: de 7:00 a.m. a 12:00 p.m.; de 12:00 p.m. a 3:00 p.m.; de 3:00 p.m. a 12:00 a.m. La identificación de cada intervalo se basó en la tasa de llegada de los pacientes al sistema, siendo el primer intervalo el que contiene el menor tiempo promedio entre llegadas, seguido por el segundo intervalo, y por último el de 3:00 p.m. a 12 a.m. La tasa de llegada es despreciable de 12 a.m. a 7 a.m. Este comportamiento es similar de lunes a viernes, teniéndose una pequeña diferencia el fin de semana, donde la mayor afluencia de pacientes es de 12:00 p.m. a 3:00 p.m. Además, la semana tomada de referencia es representativa de lo que acontece semanalmente en Emergencia Adultos.
Los días lunes se tiene mayor afluencia de pacientes, siendo menor de martes a jueves, sin embargo los viernes y sábados se tiene un pequeño incremento en el arribo de los pacientes, por último, el domingo es el día menos concurrido.

\section{Tamaño de muestra}

Para cada intervalo establecido y en cada día de la semana, se evaluó el comportamiento de los tiempos entre llegadas y la variación de éstos según el tamaño de muestra. En la Tabla N. ${ }^{\circ} 5$ se presenta un resumen del tamaño de muestra para cada intervalo de tiempo. La fórmula empleada para determinar el tamaño de muestra con un nivel de confiabilidad del $95 \%$ es $\mathrm{N}=(1.96)^{2 \star}(\mathrm{S})^{2} / \mathrm{d}^{2}$.

\section{Tiempos entre llegadas}

En la Tabla N. ${ }^{\circ} 6$ se presenta la distribución de probabilidades del tiempo entre llegadas para cada intervalo de tiempo definido. Para obtener estos resultados se tomaron los tiempos de llegada en cada horario y cada día de la semana y se analizaron mediante la prueba de Kolgomorov, con un p-valor mayor a $5 \%$ y el menor error.

Tabla $\mathbf{N}^{\circ}$ 5. Tamaño de muestra para cada intervalo de tiempo simulado.

\begin{tabular}{|c|l|c|c|c|c|}
\hline $\begin{array}{c}\text { Día de la } \\
\text { semana }\end{array}$ & \multicolumn{1}{|c|}{ Horario } & Promedio & $\begin{array}{c}\text { Desviación } \\
\text { estándar (S) }\end{array}$ & Precisión (d) & $\begin{array}{c}\text { Tamaño de } \\
\text { muestra }\end{array}$ \\
\hline \multirow{4}{*}{ Lunes } & 7:00 a.m. a 12:00 p.m & 0.93 & 0.26 & 0.0510 & 100 \\
\cline { 2 - 6 } & 12:00 p.m. a 3:00 p.m. & 1.34 & 0.20 & 0.0506 & 60 \\
\cline { 2 - 6 } & 3:00 p.m. a 12:00 a.m. & 3.34 & 0.18 & 0.0494 & 51 \\
\hline \multirow{4}{*}{ Martes } & 7:00 a.m. a 12:00 p.m & 1.83 & 0.28 & 0.0501 & 120 \\
\cline { 2 - 6 } & 12:00 p.m. a 3:00 p.m. & 2.66 & 0.21 & 0.0496 & 69 \\
\cline { 2 - 6 } & 3:00 p.m. a 12:00 a.m. & 4.06 & 0.19 & 0.0502 & 55 \\
\hline \multirow{4}{*}{ Juércoles } & 7:00 a.m. a 12:00 p.m & 1.80 & 0.20 & 0.0506 & 60 \\
\cline { 2 - 6 } & 12:00 p.m. a 3:00 p.m. & 3.41 & 0.16 & 0.0496 & 40 \\
\cline { 2 - 6 } & 3:00 p.m. a 12:00 a.m. & 5.01 & 0.17 & 0.0491 & 46 \\
\hline \multirow{4}{*}{ Viernes } & 7:00 a.m. a 12:00 p.m & 1.94 & 0.25 & 0.0505 & 94 \\
\cline { 2 - 6 } & 12:00 p.m. a 3:00 p.m. & 3.94 & 0.19 & 0.0489 & 58 \\
\cline { 2 - 6 } & 3:00 p.m. a 12:00 a.m. & 3.95 & 0.16 & 0.0502 & 39 \\
\cline { 2 - 6 } & 7:00 a.m. a 12:00 p.m & 2.08 & 0.21 & 0.0488 & 71 \\
\cline { 2 - 6 } & 12:00 p.m. a 3:00 p.m. & 2.15 & 0.21 & 0.0488 & 71 \\
\cline { 2 - 6 } 3:00 p.m. a 12:00 a.m. & 3.62 & 0.19 & 0.0498 & 56 \\
\hline \multirow{3}{*}{ Sábado } & 7:00 a.m. a 12:00 p.m & 2.62 & 0.21 & 0.0499 & 68 \\
\cline { 2 - 6 } & 12:00 p.m. a 3:00 p.m. & 2.44 & 0.16 & 0.0516 & 37 \\
\cline { 2 - 6 } & 3:00 p.m. a 12:00 a.m. & 3.49 & 0.18 & 0.0489 & 52 \\
\hline \multirow{3}{*}{ Domingo } & 7:00 a.m. a 12:00 p.m & 4.11 & 0.21 & 0.0499 & 68 \\
\cline { 2 - 6 } & 12:00 p.m. a 3:00 p.m. & 2.34 & 0.15 & 0.0504 & 34 \\
\cline { 2 - 6 } & 3:00 p.m. a 12:00 a.m. & 4.36 & 0.21 & 0.0499 & 68 \\
\hline
\end{tabular}

Fuente: Elaboración propia 
Tabla N. 6. Distribución de probabilidades del tiempo entre llegadas.

\begin{tabular}{|c|c|c|}
\hline Día de la semana & Horario & Distribución de probabilidad \\
\hline \multirow{3}{*}{ Lunes } & 7:00 a.m. a $12: 00$ p.m & $-0.001+$ EXPO (0.93) \\
\hline & 12:00 p.m. a 3:00 p.m. & TRIA(-0.001, 0.0695, 3.94) \\
\hline & 3:00 p.m. a 12:00 a.m. & $-0.001+$ EXPO (3.34) \\
\hline \multirow{3}{*}{ Martes } & 7:00 a.m. a $12: 00$ p.m & $-0.001+$ WEIB $(1.92,1,15)$ \\
\hline & 12:00 p.m. a 3:00 p.m. & $-0.001+$ WEIB $(2.72,1.06)$ \\
\hline & 3:00 p.m. a 12:00 a.m. & $-0.001+$ EXPO (4.06) \\
\hline \multirow{3}{*}{ Miércoles } & 7:00 a.m. a $12: 00$ p.m & $-0.001+$ WEIB $(1.74,0.931)$ \\
\hline & 12:00 p.m. a 3:00 p.m. & $-0.001+$ EXPO (3.41) \\
\hline & 3:00 p.m. a 12:00 a.m. & $-0.001+13^{*} \operatorname{BETA}(0.918,1.46)$ \\
\hline \multirow{3}{*}{ Jueves } & 7:00 a.m. a $12: 00$ p.m & $-0.001+\operatorname{LOGN}(2.52,5.64)$ \\
\hline & 12:00 p.m. a 3:00 p.m. & $-0.001+$ WEIB $(4.15,1.17)$ \\
\hline & 3:00 p.m. a $12: 00$ a.m. & EXPO(3.95) \\
\hline \multirow{3}{*}{ Viernes } & 7:00 a.m. a $12: 00$ p.m & $-0.001+$ EXPO (2.08) \\
\hline & 12:00 p.m. a 3:00 p.m. & $-0.001+$ WEIB $(2.23,1.11)$ \\
\hline & 3:00 p.m. a $12: 00$ a.m. & $-0.001+11^{*} \operatorname{BETA}(0.713,1.52)$ \\
\hline \multirow{3}{*}{ Sábado } & 7:00 a.m. a $12: 00$ p.m & $-0.001+13^{*} \operatorname{BETA}(0.838,3.22)$ \\
\hline & 12:00 p.m. a 3:00 p.m. & $-0.001+$ EXPO $(2.44)$ \\
\hline & 3:00 p.m. a $12: 00$ a.m. & $-0.001+$ WEIB $(3.36,0.925)$ \\
\hline \multirow{3}{*}{ Domingo } & 7:00 a.m. a $12: 00$ p.m & $-0.001+$ EXPO (4.11) \\
\hline & 12:00 p.m. a 3:00 p.m. & $-0.001+$ EXPO (2.34) \\
\hline & 3:00 p.m. a $12: 00$ a.m. & $-0.001+$ WEIB $(4.08,0.869)$ \\
\hline
\end{tabular}

Fuente: Elaboración propia

Tiempos de espera

En el modelo de simulación se evaluarán los tiempos de espera generados en cada ambiente, sin embargo en los ambientes donde se realizan los análisis clínicos y los rayos $\mathrm{X}$, no se consideran tiempos de espera, debido a que el tiempo de atención incluye el tiempo de espera previo.

\section{Tiempos de servicio}

En la Tabla N ${ }^{\circ} 7$ se presenta la distribución de probabilidades de la duración del servicio. Para obtener estos resultados se tomaron los tiempos de atención para cada tópico y se analizaron mediante la prueba de Kolgomorov con un p-valor mayor a $5 \%$ y el menor error.

Tabla N. ${ }^{\circ}$ 7. Distribución de probabilidades de la duración del servicio.

\begin{tabular}{|l|l|}
\hline \multicolumn{1}{|c|}{ Proceso } & \multicolumn{1}{c|}{ Distribución de probabilidad } \\
\hline Atención en Triaje & $\operatorname{EXPO}(0.961)$ \\
\hline Atención en Admisión & $\operatorname{LOGN}(2.22,2.01)$ \\
\hline Atención en Medicina & $2+27^{*} \mathrm{BETA}(0.68,1.59)$ \\
\hline Atención en Cirugía & $2+\operatorname{WEIB}(9.26,1.29)$ \\
\hline Atención en Traumatología & $1+\operatorname{GAMM}(3.54,1.7)$ \\
\hline Análisis en Laboratorio & $1+\operatorname{EXPO}(2.43)$ \\
\hline Atención en Digitación & $\operatorname{LOGN}(2.35,3.32)$ \\
\hline Atención en Farmacia & $\operatorname{LOGN}(1,0.874)$ \\
\hline Resultado de otros análisis & $62+437^{*} \mathrm{BETA}(1.16,2.93)$ \\
\hline Resultado de Rayos $\mathrm{X}$ & $24.5+26^{*} \mathrm{BETA}(0.799,1.13)$ \\
\hline
\end{tabular}

Fuente: Elaboración propia
Supuestos considerados en el modelo

En el diseño del modelo se han considerado los siguientes supuestos:

a) La atención del paciente dura hasta el momento en que el doctor obtiene un diagnóstico y prescribe la medicina al paciente.

b) Se considera que el paciente sale del sistema en cualquiera de los siguientes casos:

- Se interna al paciente en el hospital.

- Se mantiene al paciente en sala de observación.

- Se atiende al paciente y después este se retira.

c) Al llegar un paciente de shock o trauma esperará su turno para ser atendido en la sala de Triaje, es decir, no interrumpe la atención de otro paciente, sin embargo, se le ha considerado la mayor prioridad.

d) Un paciente se atiende en un solo tópico.

e) El tiempo de desplazamiento de las personas que toman la muestra de sangre hacia el laboratorio y su regreso a la unidad es constante.

f) El tiempo que un paciente Tipo 2 requiere para asimilar un medicamento es constante. 
g) Los pacientes esperan hasta ser atendidos, no abandonan el sistema por espera excesiva.

h) Se trabajará el modelo como un sistema terminal. El sistema comienza a simularse el día lunes a las 7:00 a.m. con cero pacientes y finaliza el lunes siguiente a la misma hora también con cero pacientes (el domingo es el día menos concurrido). Se simulan 10,080 minutos (una semana).

La simulación se realizó empleando el software Arena, se efectuaron ochenta réplicas de la simulación para obtenerse errores máximos de $6 \%$.

\section{RESULTADOS DEL SISTEMA ACTUAL}

En la Tabla $N .^{\circ} 8$ se muestran los tiempos de permanencia en el sistema para cada tipo de paciente.

Como se aprecia en la Tabla $\mathrm{N}^{\circ} 8$, los pacientes tipo 1, 3, 7 y 11 son los que permanecen más tiempo en el sistema.

Los pacientes tipo 7 y 11 son aquellos que ingresan a los tópicos de medicina y cirugía, con resultados de análisis. El tiempo obtenido para estos dos tipos de pacientes es coherente con el tiempo promedio que se demora el laboratorio en obtener los resultados de análisis (180 minutos). Falta considerar el tiempo de atención en el tópico.

Los pacientes tipo 1 y 3 son pacientes de alivio que si bien no requieren de ningún tipo de análisis, se atienden en el tópico de medicina que es el más concurrido. Estos pacientes tienen prioridad IV.

Los pacientes tipo 15 son los que menos tiempo permanecen en el sistema debido a que tienen prioridad I.

Tabla N..$^{\circ}$ 8. Tiempos de permanencia en el sistema para cada tipo de paciente

\begin{tabular}{|c|c|}
\hline $\begin{array}{c}\text { Tipo de } \\
\text { paciente }\end{array}$ & $\begin{array}{c}\text { Tiempo de permanencia en el } \\
\text { sistema (minutos) }\end{array}$ \\
\hline 1 & 430 \\
\hline 2, luego 3 & 515 \\
\hline 4 & 88 \\
\hline $\begin{array}{c}\text { 5, luego 7 } \\
\text { 6, luego 7 }\end{array}$ & 325 \\
\hline 8 & 16 \\
\hline $\begin{array}{c}\text { 9, luego 11 } \\
\text { 10, luego 11 }\end{array}$ & 232 \\
\hline 12 & 18 \\
\hline 13, luego 14 & 64 \\
\hline 15 & 3 \\
\hline
\end{tabular}

Fuente: Elaboración propia
En la Tabla N. ${ }^{\circ} 9$ se muestran los porcentajes de utilización en cada unidad de servicio.

Tabla N. 9. Porcentajes de utilización de las unidades de servicio.

\begin{tabular}{|l|c|}
\hline \multicolumn{1}{|c|}{ Ambiente } & Utilización \\
\hline Sala de reposo & 0.000 \\
\hline Triaje & 0.169 \\
\hline Admisión & 0.246 \\
\hline Medicina & 0.665 \\
\hline Cirugía & 0.211 \\
\hline Traumatología & 0.240 \\
\hline Laboratorio & 0.121 \\
\hline Digitación en Análisis & 0.223 \\
\hline Farmacia & 0.108 \\
\hline Shock-Trauma & 0.052 \\
\hline
\end{tabular}

Fuente: Elaboración propia

El tópico de Medicina tiene la mayor afluencia de pacientes, sin embargo su utilización es de $66.5 \%$, lo que puede deberse a una mala distribución del personal médico en los tres turnos.

Los tópicos de Cirugía y Traumatología, tienen utilizaciones de $21.1 \%$ y $24.0 \%$ respectivamente, esto hace suponer que hay excesivo personal médico asignado.

Actualmente, se tiene la misma cantidad de médicos por turno durante toda la semana. Tres médicos atienden por turno en el tópico de Medicina, igualmente, tres atienden por turno en el tópico de Cirugía, y uno atiende por turno en el tópico de Traumatología.

Los demás ambientes de atención (Digitación, Farmacia, Admisión y Análisis en Laboratorio) tienen baja utilización, sin embargo no se considerarán ambientes críticos, debido a que prestan servicios a otras unidades del hospital, y los resultados obtenidos sólo corresponden a la zona de Emergencia Adultos.

\section{PROPUESTA DE SOLUCIÓN}

Se evaluaron siete propuestas de solución que se resumen en la Tabla $\mathrm{N} .{ }^{\circ} 10$. Los tiempos de permanencia en el sistema de los pacientes tipo 8, 12, 14 y 15 no difieren significativamente de la situación actual ( \pm 1 minuto). En todas las propuestas, un médico por turno atenderá en el tópico de Traumatología. 
Tabla N. ${ }^{\circ}$ 10. Resumen de las propuestas de solución para Emergencia Adultos.

\begin{tabular}{|c|c|c|}
\hline $\begin{array}{l}\text { Número de } \\
\text { propuesta }\end{array}$ & Descripción & $\begin{array}{l}\text { Tiempo de permanencia en el } \\
\text { sistema }\end{array}$ \\
\hline 1 & $\begin{array}{l}\text { En el tópico de Medicina atenderán cuatro } \\
\text { médicos de 7:00 a.m. a 7:00 p.m. y dos } \\
\text { médicos el resto del día. } \\
\text { En el tópico de Cirugía atenderán tres } \\
\text { médicos en cada turno. }\end{array}$ & $\begin{array}{l}301 \text { minutos el paciente tipo } 1 \\
\text { (reducción de } 129 \text { minutos) } \\
369 \text { minutos el paciente tipo } 3 \\
\text { (reducción de } 146 \text { minutos) } \\
57 \text { minutos el paciente tipo } 4 \\
\text { (reducción de } 31 \text { minutos) } \\
294 \text { minutos el paciente tipo } 7 \\
\text { (reducción de } 31 \text { minutos) } \\
238 \text { minutos el paciente tipo } 11 \\
\text { (aumento de } 6 \text { minutos) }\end{array}$ \\
\hline 2 & $\begin{array}{l}\text { En el tópico de Medicina atenderán cuatro } \\
\text { médicos de 7:00 a.m. a 7:00 p.m. y dos } \\
\text { médicos el resto del día. } \\
\text { En el tópico de Cirugía atenderán cuatro } \\
\text { médicos de 7:00 a.m. a 7:00 p.m. y dos } \\
\text { médicos el resto del día. }\end{array}$ & $\begin{array}{l}301 \text { minutos el paciente tipo } 1 \\
\text { (reducción de } 129 \text { minutos) } \\
365 \text { minutos el paciente tipo } 3 \\
\text { (reducción de } 150 \text { minutos) } \\
58 \text { minutos el paciente tipo } 4 \\
\text { (reducción de } 30 \text { minutos) } \\
296 \text { minutos el paciente tipo } 7 \\
\text { (reducción de } 29 \text { minutos) } \\
236 \text { minutos el paciente tipo } 11 \\
\text { (aumento de } 4 \text { minutos) }\end{array}$ \\
\hline 3 & $\begin{array}{l}\text { En el tópico de Medicina atenderán cuatro } \\
\text { médicos de 7:00 a.m. a 7:00 p.m. y dos } \\
\text { médicos el resto del día. } \\
\text { En el tópico de Cirugía atenderán tres } \\
\text { médicos en cada turno. } \\
\text { Reducir en } 30 \text { minutos la obtención de los } \\
\text { resultados de Análisis en Laboratorio. }\end{array}$ & $\begin{array}{l}319 \text { minutos el paciente tipo } 1 \\
\text { (reducción de } 111 \text { minutos) } \\
406 \text { minutos el paciente tipo } 3 \\
\text { (reducción de } 109 \text { minutos) } \\
62 \text { minutos el paciente tipo } 4 \\
\text { (reducción de } 26 \text { minutos) } \\
270 \text { minutos el paciente tipo } 7 \\
\text { (reducción de } 55 \text { minutos) } \\
208 \text { minutos el paciente tipo } 11 \\
\text { (reducción de } 24 \text { minutos) }\end{array}$ \\
\hline 4 & $\begin{array}{l}\text { En el tópico de Medicina atenderán tres } \\
\text { médicos en cada turno. } \\
\text { En el tópico de Cirugía atenderán tres } \\
\text { médicos en cada turno. } \\
\text { En el segundo turno los médicos de } \\
\text { la Sala de Triaje apoyan al tópico de } \\
\text { Medicina, atendiendo también a los } \\
\text { pacientes de alivio. }\end{array}$ & $\begin{array}{l}403 \text { minutos el paciente tipo } 1 \\
\text { (reducción de } 27 \text { minutos) } \\
488 \text { minutos el paciente tipo } 3 \\
\text { (reducción de } 27 \text { minutos) } \\
86 \text { minutos el paciente tipo } 4 \\
\text { (reducción de } 2 \text { minutos) } \\
324 \text { minutos el paciente tipo } 7 \\
\text { (reducción de } 1 \text { minuto) } \\
238 \text { minutos el paciente tipo } 11 \\
\text { (aumento de } 6 \text { minutos) }\end{array}$ \\
\hline 5 & $\begin{array}{l}\text { En el tópico de medicina atenderán cuatro } \\
\text { médicos de 7:00 a.m. a 7:00 p.m. y un } \\
\text { médico el resto del día. } \\
\text { En el tópico de cirugía atenderán tres } \\
\text { médicos en cada turno. }\end{array}$ & $\begin{array}{l}616 \text { minutos el paciente tipo } 1 \\
\text { (aumento de } 186 \text { minutos) } \\
727 \text { minutos el paciente tipo } 3 \\
\text { (aumento de } 212 \text { minutos) } \\
110 \text { minutos el paciente tipo } 4 \\
\text { (aumento de } 22 \text { minutos) } \\
352 \text { minutos el paciente tipo } 7 \\
\text { (aumento de } 27 \text { minutos) } \\
240 \text { minutos el paciente tipo } 11 \\
\text { (aumento de } 8 \text { minutos) }\end{array}$ \\
\hline
\end{tabular}


Tabla N. ${ }^{\circ}$ 10. Resumen de las propuestas de solución para Emergencia Adultos.

\begin{tabular}{|c|c|c|}
\hline $\begin{array}{l}\text { Número de } \\
\text { propuesta }\end{array}$ & Descripción & $\begin{array}{l}\text { Tiempo de permanencia en el } \\
\text { sistema }\end{array}$ \\
\hline 6 & $\begin{array}{l}\text { En el tópico de Medicina atenderán cinco } \\
\text { médicos de 7:00 a.m. a 7:00 p.m. y un } \\
\text { médico el resto del día. } \\
\text { En el tópico de Cirugía atenderán tres } \\
\text { médicos en cada turno. }\end{array}$ & $\begin{array}{l}251 \text { minutos el paciente tipo } 1 \\
\text { (reducción de } 179 \text { minutos) } \\
339 \text { minutos el paciente tipo } 3 \\
\text { (reducción de } 176 \text { minutos) } \\
73 \text { minutos el paciente tipo } 4 \\
\text { (reducción de } 15 \text { minutos) } \\
315 \text { minutos el paciente tipo } 7 \\
\text { (reducción de } 10 \text { minutos) } \\
240 \text { minutos el paciente tipo } 11 \\
\text { (reducción de } 8 \text { minutos) }\end{array}$ \\
\hline 7 & $\begin{array}{l}\text { En el tópico de Medicina atenderán dos } \\
\text { médicos en cada turno (no atienden a los } \\
\text { pacientes de alivio). } \\
\text { En el tópico de Cirugía atenderán tres } \\
\text { médicos en cada turno. } \\
\text { En un ambiente separado un médico en } \\
\text { cada turno atenderá a los pacientes de } \\
\text { alivio. }\end{array}$ & $\begin{array}{l}26 \text { minutos el paciente tipo } 1 \\
\text { (reducción de } 404 \text { minutos) } \\
70 \text { minutos el paciente tipo } 3 \\
\text { (reducción de } 445 \text { minutos) } \\
383 \text { minutos el paciente tipo } 4 \\
\text { (aumento de } 295 \text { minutos) } \\
624 \text { minutos el paciente tipo } 7 \\
\text { (aumento de } 299 \text { minutos) } \\
231 \text { minutos el paciente tipo } 11 \\
\text { (disminución de } 1 \text { minuto) }\end{array}$ \\
\hline
\end{tabular}

Fuente: Elaboración propia

\section{CONCLUSIONES}

Las principales conclusiones de la investigación son:

- La utilización de los médicos es menor al $70 \%$, lo que indica que no es recomendable contratar más personal para disminuir los tiempos de atención.

- Se logra una reducción considerable en el tiempo de permanencia de los pacientes que son atendidos en el tópico de medicina asignando a cuatro médicos en el horario de 7:00 a.m. a las 7:00 p.m. y dos médicos de 7:00 p.m. a 7:00 a.m.

- En el sistema actual los mayores tiempos de permanencia lo tienen los pacientes tipo 3 (paciente de alivio que requiere una segunda revisión), siendo este tiempo de 515 minutos ( 8.58 horas), seguidos por los pacientes tipo 1 (pacientes de alivio que no requieren segunda revisión) con 430 minutos ( 7.17 horas) y por los pacientes tipo 7 (paciente de medicina con resultados de análisis) con 325 minutos (5.42 horas). Con la propuesta 1 sus tiempos se redujeron a 369 , 301 y 294 minutos $(6.15,5.02$ y 4.90 horas) respectivamente.

\section{RECOMENDACIONES}

La principal recomendación es:

- Evaluar la posibilidad de adquirir una aplicación informática que permita integrar la información de los pacientes de emergencia generada en los diferentes ambientes del área Emergencia Adultos (Digitación, Laboratorios, Farmacia, Rayos $\mathrm{X}$, etc.)

\section{REFERENCIAS BIBLIOGRÁFICAS}

[1] Delgado K.C. (2007), Diagnóstico y Propuestas de Mejora del Sistema de Atención a Pacientes de Emergencia Adultos de un Hospital Público Utilizando Simulación Discreta. Tesis (Ingeniera Industrial), Pontificia Universidad Católica del Perú, Facultad de Ciencias e Ingeniería, Lima, Perú.

[2] Fitzsimmons, J. A. Y FITZSIMMONS, M. J. (2008). Service Management: Operations, Strategy and Information Technology. 6ta. ed. McGraw-Hill/ Irwin.

[3] Kelton, W.D.; Sadowski, R.P. And Sturrock, D.T. (2008), Simulación con Software Arena. Cuarta ed. McGraw Hill.

[4] Torres, P. (2010), Simulación de Sistemas con el software Arena. Fondo Editorial, Universidad de Lima. 